\title{
Mitogen-activated protein kinase signaling pathway in oral cancer (Review)
}

\author{
QIAN PENG, ZHIYUAN DENG, HAO PAN, LIQUN GU, OUSHENG LIU and ZHANGUI TANG \\ Xiangya Stomatological Hospital, Central South University, Changsha, Hunan 410008, P.R. China
}

Received July 4, 2016; Accepted August 10, 2017

DOI: $10.3892 / \mathrm{ol} .2017 .7491$

\begin{abstract}
The mitogen-activated protein kinase (MAPK) signaling pathway is associated with tumor cell proliferation, differentiation, apoptosis, angiogenesis, invasion and metastasis. The present review assesses the involvement of the MAPK signaling pathway in oral cancer progression and invasion based on analysis of individual sub-pathways and their mechanisms of action. The regulation of this pathway for targeted oral cancer therapy is explored and the challenges confronting this, as well as corresponding potential solutions, are discussed. Exploring this pathway with an emphasis on its components, subfamilies, sub-pathways, interactions with other pathways and clinical practice modes may improve oral cancer treatment.
\end{abstract}

\section{Contents}

1. Introduction

2. MAPK signaling sub-pathways

3. Action mechanisms of the MAPK signaling pathway in oral cancer

4. Regulation of the MAPK signaling pathway for targeted oral cancer therapy

5. Discussion

6. Conclusions

\section{Introduction}

Head and neck malignant carcinoma is a commonly diagnosed cancer and its incidence has risen over the past decade (1). Oral squamous cell carcinoma (OSCC) is an important oral malignancy, accounting for $>90 \%$ of head and neck malignant

Correspondence to: Professor Zhangui Tang, Xiangya Stomatological Hospital, Central South University, 72 Xiangya Road, Changsha, Hunan 410008, P.R. China

E-mail: zhgtang@csu.edu.cn

Key words: mitogen-activated protein kinase signaling pathway, oral cancer, oral squamous cell carcinoma, kinase, therapeutic targets, inhibitor, treatment carcinoma cases (2). It has a 5-year survival rate of $\sim 50 \%$ (3), due to delayed diagnosis, disease recurrence, distant metastasis and therapeutic resistance. At present, multiple attempts are being made to develop efficient targeted therapies, which often involve the use of various inhibitors for improving treatment due to their special advantages, such as low cytotoxicity, strong specificity and long duration of action.

Targeted therapies aim at controlling the signaling pathways through which cell survival and death are regulated. Multiple signaling pathways may function in oncogenesis and in the growth of oral malignancies (4,5). Among these is the mitogen activated protein kinase (MAPK) signaling pathway, which regulates the expression of a large number of proteins involved in the control of cell proliferation, differentiation and apoptosis $(6,7)$, and remains a focus of investigation for therapeutic targeting, along with the development of appropriate inhibitors (8). These inhibitors have been demonstrated to be effective for the treatment of oral cancer and other malignancies, including human colon cancer, breast cancer and lung cancer (9-12). Certain inhibitors, which have been examined in various clinical trials, are being used to treat patients with oral cancer and are eliciting promising results. The purpose of the present review is to provide an update on the involvement of the MAPK pathway in oral cancer progression and invasion, and to review the progress made in the development of pharmacologic inhibitors to regulate the MAPK signaling pathway, in order to improve oral cancer treatment.

\section{MAPK signaling sub-pathways}

The MAPK signaling pathway is comprised of four sub-pathways, namely the extracellular signal-regulated kinase (ERK)1/2) sub-pathway, the c-Jun N-terminal kinase (JNK) sub-pathway, the p38 sub-pathway and the ERK5 sub-pathway (13). These sub-pathways correspond to the ERK1/2, JNK1/2/3, p38 $\alpha / \beta / \gamma / \delta$ and ERK5 subfamilies of MAPK, respectively, and are a chain of proteins that transduce various extracellular signals to the nucleus, controlling gene expression through transcriptional factors (14). By regulating protein activities through signaling cascades that consist of MAPK kinase kinase (MAPKKK), MAPK kinase and MAPK (15), these sub-pathways impact tumor cell proliferation, differentiation and survival $(16,17)$, as presented in Fig. 1.

The ERK1/2 sub-pathway has received the most attention thus far (18-20). It has 3 important components: Ras, Raf and 
MEK. As shown in Fig. 1, in response to the binding of growth factors and hormones to cell surface receptors (21), the level of Ras-guanosine triphosphate (GTP) increases in cells, which in turn promotes kinase activation. The GTP-bound forms of Ras directly bind and thus recruit cytosolic dimers of the Raf kinases to the plasma membrane (22). Once localized at the membrane, Raf is activated through phosphorylation by other kinases or autophosphorylation. It then phosphorylates and activates MEK, which subsequently induces the phosphorylation and activation of ERK. The activated ERK1/2 dissociates from the Ras-Raf-MEK-ERK1/2 complex and phosphorylates a number of cytoskeletal proteins, kinases, and transcription factors, including nuclear factor (NF)- $\mathrm{B}$, AP-1, ETS-1, c-Myc, and members of the signal transducer and activator of transcription family $(23,24)$. The functional consequences of substrate-level phosphorylation by ERK1/2 include changes in cellular motility and gene expression that promote proliferation, differentiation, cellular survival, immortalization and angiogenesis (25).

The JNK sub-pathway is associated with activation of MAPKKK [mitogen activated protein kinase kinase kinase (MEKK)1/2/3/4], a response induced by a number of environmental stresses (including osmotic stress, UV light, heat shock and growth factor withdrawal) and cytokines [including tumor necrosis factor (TNF)- $\alpha$ and interleukin (IL)- $\beta$ ] (26). The activated MEKK1/2/3/4 in the sub-pathway phosphorylates a series of proteins in the following order: Mitogen activated protein kinase kinase $(\mathrm{MKK}) 4 / 7 \rightarrow \mathrm{JNK} 1 / 2 / 3 \rightarrow$ c-Jun, activating transcription factor (ATF)-2, and ELK1, ETS transcription factor. The sub-pathway contributes to cell growth, differentiation, apoptosis and survival through stress/cytokine-sensitive responses (27).

Similar to the JNK sub-pathway, the p38 sub-pathway is associated with the response of MAPKKK [mixed lineage kinases (MLKs), mitogen activated protein kinase kinase kinase 7 (TAK-1) and mitogen activated protein kinase kinase kinase 5 (ASK-1)] caused by stress and pro-inflammatory cytokines, including IL-1 and TNF- $\alpha$ (28). The activated MLKs, TAK-1 and ASK-1 in this sub-pathway phosphorylate and activate various kinases sequentially, from MLKs, TAK-1, ASK-1 to MKK3/6 to the four p38 isoforms (p38 $\alpha / \beta / \gamma / \delta)$. The activated p38 isoforms then activate the substrates ATF-2, cAMP response element-binding protein, DNA damage inducible transcript 3 and myocyte enhancer factor (MEF)2C. Cell differentiation, apoptosis, survival and inflammation are induced by this pathway (29).

In comparison with the other sub-pathways, the ERK5 sub-pathway has beenless intensively investigated (30).It is associated with a variety of stimuli, including mitogens (for example, epithermal growth factor) and cellular stresses (including oxidation and osmotic stress) (31). Its activation is consistent with the cascade MEKK2/3 $\rightarrow$ MKK $5 \rightarrow$ ERK $5 \rightarrow$ MEF2A/C/D AP-1 transcription factor subunit (C-Fos) and Fos related antigen-1, and the ERK5 sub-pathway regulates cell growth, differentiation and survival $(32,33)$.

\section{Action mechanisms of the MAPK signaling pathway in oral cancer}

The MAPK signaling pathway is involved in various cellular responses, including cell proliferation, apoptosis,

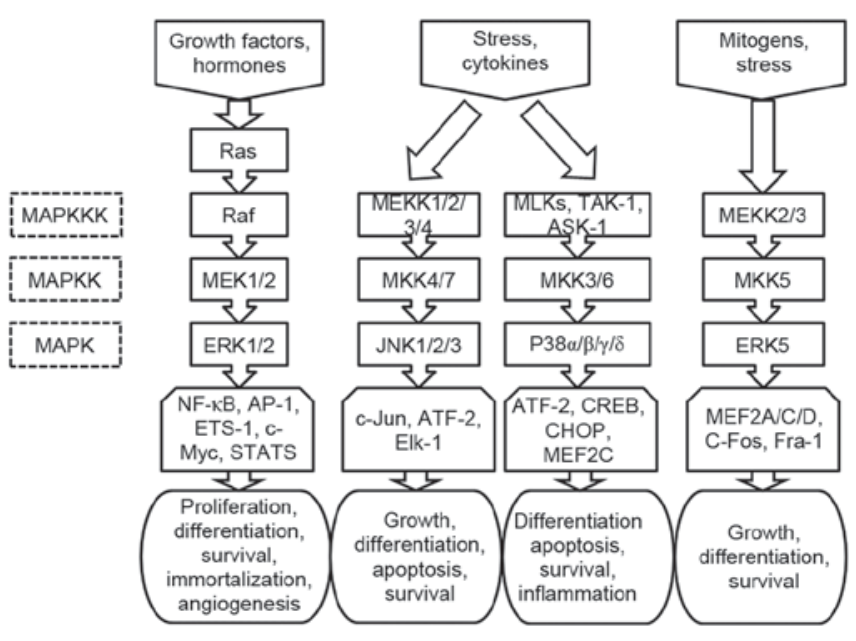

Figure 1. Schematic illustration of MAPK signaling pathway, where each MAPK phosphorylates several transcriptional factors, regulating protein expression.

angiogenesis, cell migration and metastasis. The deregulation of MAPK signaling leads to inappropriate responses, induced through abnormal gene expression. Further elucidation of the mechanisms underlying the action of proteins that regulate MAPK signaling and of their expression patterns in oral cancer may provide novel insights into the signal transduction mechanisms in cell cycle progression and malignant transformation. A large number of proteins serve as targets that act on the oncogenesis and growth of oral tumor cells. These targets include angiopoietin-like 3, annexin A10, SH3 domain containing kinase binding protein 1, quaking 5, epidermal growth factor receptor, fibroblast activation protein, parathyroid hormone-related protein and 70-kDa ribosomal S6 kinase (34-41). Their functions and molecular mechanisms in the MAPK signaling pathway that concern oral cancer are summarized in Table I.

Promoting tumor cell proliferation. Within the MAPK signaling pathway, two main mechanisms are responsible for promoting tumor cell proliferation. The primary mechanism is associated with early gene-encoded c-Jun and C-Fos proteins, which constitute transcription factor AP-1 in the form of a heterodimer or homodimer following MAPK phosphorylation; for example, ERK phosphorylation (18). These factors then combine with corresponding promoter regions of DNA and upregulate the expression of cyclin D1, which promotes the progression of the cell cycle from the G1 phase to the $\mathrm{S}$ phase and accelerates cell proliferation. The other mechanism is attributed to the function of activated MAPK, which suppresses expression of cell cycle inhibitory proteins including $\mathrm{p} 27^{\mathrm{KIP}}$, attenuating the inhibition of the cell cycle (42).

Inhibiting tumor cell apoptosis. There are three main mechanisms of the MAPK signaling pathway that inhibit tumor cell apoptosis. Firstly, the signaling pathway may directly suppress the activity of the apoptosis end effector, caspase-3, which inhibits hydrolysis of tubulin to maintain the integrity of its spindle body. This function prevents cell apoptosis from being induced by various stimuli. Secondly, the pathway may 
indirectly suppress the activity of caspase- 3 by activating the inhibitors of apoptosis molecules, including inhibitor of apoptosis and B cell lymphoma-2 family proteins $(43,44)$. Thirdly, the pathway may block the release of mitochondrial cytochrome $\mathrm{C}$ and interfere with the activation process of upstream caspase- 9 , indirectly decreasing the activity of downstream caspase-3 (45).

Promoting tumor angiogenesis. Tumor angiogenesis not only provides nutrition for increasingly active tumor cell division, but is also involved in the outward expansion of the tumor. A number of vascular growth factors upregulate the expression level of vascular endothelial growth factor via activation of the MAPK signaling pathway, resulting in angiogenesis (38).

Inducing tumor invasion and metastasis. The process of tumor invasion relies on the precise coordination of extracellular matrix adhesion and hydrolysis in association with two members of the matrix metalloproteinase (MMP) family; namely MMP-2 and MMP-9. The expression of MMPs is primarily controlled by MAPK and other signaling pathways, including the Janus kinase (JAK)/signal transducer and activator of transcription (STAT) pathway (46). This has been validated by a number of studies in which the inhibitory functions of anticancer drugs on tumor invasion and metastasis were demonstrated to occur by downregulating the MAPK signaling pathway, which resulted in reduced expressions of MMPs $(47,48)$.

\section{Regulation of the MAPK signaling pathway for targeted oral cancer therapy}

The expression levels of the component proteins of the MAPK signaling pathway tend to increase in oral cancer (49). Activation of the MAPK signaling pathway is known to cause the transformation of normal cells to tumor phenotype (6). Thus, inhibition of this pathway may enable restoration of the tumor cells to the untransformed phenotype in vitro, thereby inhibiting the growth of the tumor in the body $(50,51)$. Theoretically, intervention targeting any component in the MAPK signaling pathway has the potential to arrest tumor growth. For this reason, multiple inhibitors have been developed to target different components of the pathway (52-55). These inhibitors serve as promising, effective antineoplastic agents for the treatment of malignant oral tumors, and are characterized by low cytotoxicity, strong specificity, a long duration of action, high solubility and metabolic stability, all of which contribute to their anti-tumor effects. At present, certain inhibitors have been used in phase I or phase II clinical trials and achieved promising effects (55).

These inhibitors may inhibit oral tumor cell proliferation by blocking the activation of corresponding target molecules of the MAPK signaling pathway. They include epigallocatechin-3-gallate, aliphatic acetogenin, galanin receptor 1 , and S-allylcysteine (56-59). Certain inhibitors, including pterostilbene, astaxanthin and sulfasalazine (SSZ) (60-62), may also induce tumor cell apoptosis. Other inhibitors include those that have the potential to inhibit either oral tumor angiogenesis (for example, cetuximab) (63) or tumor invasion and metastasis (for example, vinculin, phenethyl isothiocyanate, cardiotoxin
III and resveratrol) (64-67). Their characteristics are listed in Table II.

Besides the aforementioned inhibitors, it is worth noting that certain inhibitors may induce autophagic cell death via activation of the MAPK signaling pathway. For example, high-concentration SSZ was revealed to initially trigger autophagy, which then induced apoptosis in HSC- 4 cells by activating the ERK1/2 sub-pathway. Therefore, SSZ may have the potential to treat oral cancer (62).

\section{Discussion}

Although a number of genetic and experimental observations have validated that inhibitors of MAPK cascades would act as effective antineoplastic agents for the treatment of oral cancer with approved performance, there are a few of remaining challenges and questions to be resolved.

First, mutated members of the cascade, including Ras and Raf, are expected to serve as inhibitory targets in the MAPK pathway $(68,69)$, but their functions have not been sufficiently illustrated. The most commonly observed mutations are those that arise in the Ras genes, including H-Ras, N-Ras and K-Ras, with corresponding mutations being detected in $>30 \%$ of patients with OSCC in Southeast Asia, primarily caused by chewing tobacco (70). These Ras genes have the potential to regulate the MAPK and phosphoinositide 3-kinase $(\mathrm{PI} 3 \mathrm{~K}) /$ protein kinase B (Akt) pathways, and a Ras mutation may therefore activate both of them. As for Raf, its activity in certain cell types is negatively regulated by Akt, revealing the presence of cross-talk between the two pathways (71). Activating mutations in the B-Raf gene (one of the three Raf genes, which include A-Raf, B-Raf and C-Raf) were also identified in oral cancer (19,72). Unlike mutation of Ras, mutation of B-Raf only causes activation of the MAPK signaling pathway. It functions independently in promoting the engagement of the MAPK cascade (73). These findings confirm the complicated functions of the targets in the MAPK pathway, indicating that different inhibitors of the same target and drugs for different targets within the same pathway may demonstrate marked differences in effectiveness, depending on tumor type and mutational status. Hence, improved understanding of their biochemical properties or modes of action, along with improvements in their pharmacologic profiles, will enable more optimal treatment of oral cancers through the development of more effective therapies.

Secondly, inhibiting only a single subfamily or sub-pathway has been revealed to be insufficient to promote apoptosis or arrest growth to control oral cancer, due to a potential compensation effect of alternative subfamilies or sub-pathways (13). The occurrence of most oral cancer is associated with dysregulation of multiple subfamilies and sub-pathways. Furthermore, certain sub-pathways, including the ERK5 sub-pathway, have not been sufficiently explored and their cross-functions remain unclear. From this perspective, clearly identifying the functions and interactions of these signaling transduction sub-pathways is critical to inducing tumor cell apoptosis and overcoming tumor resistance. This is the clinical need that must be satisfied in order to improve treatment for oral cancer, and therefore warrants additional attention. 


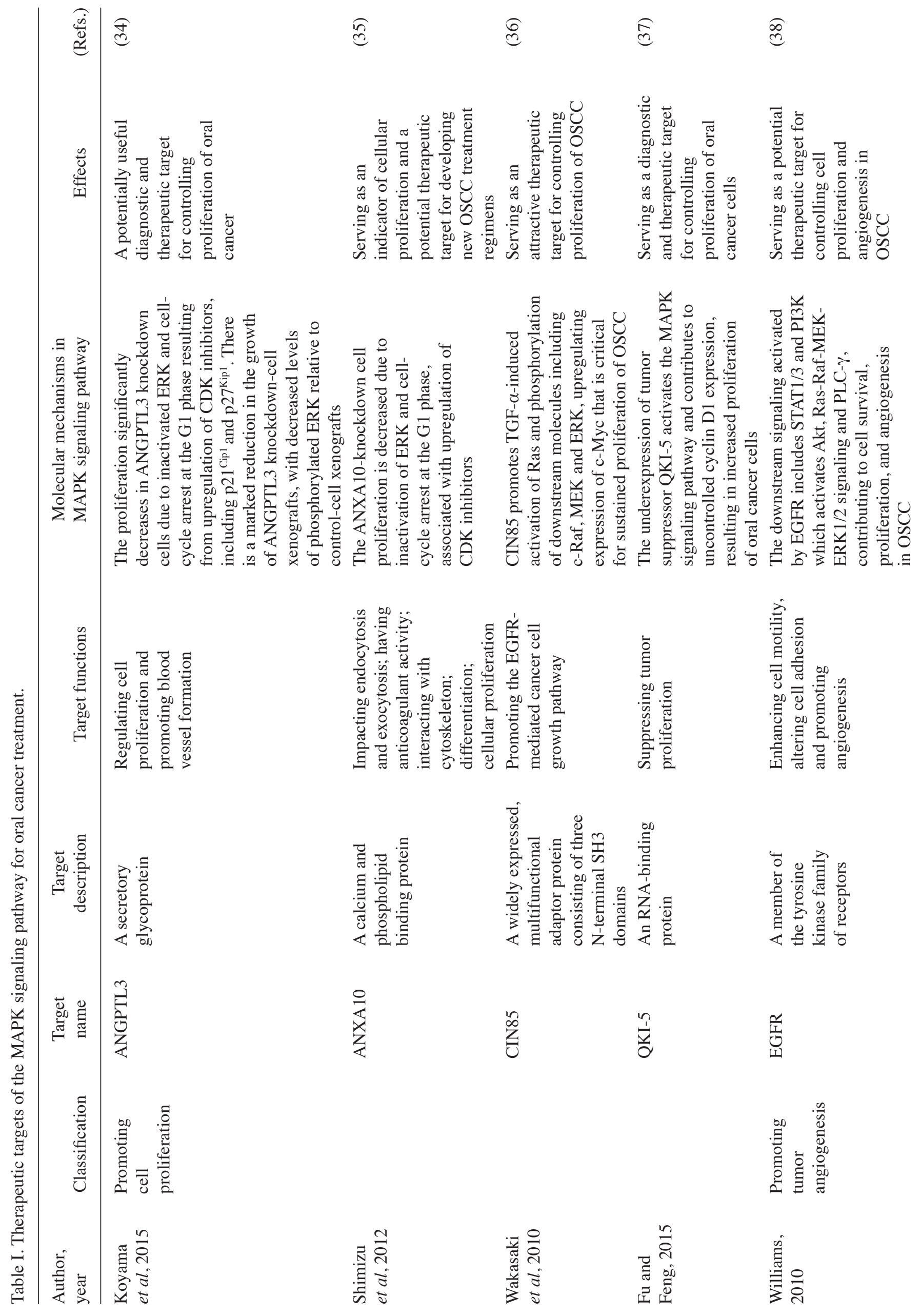




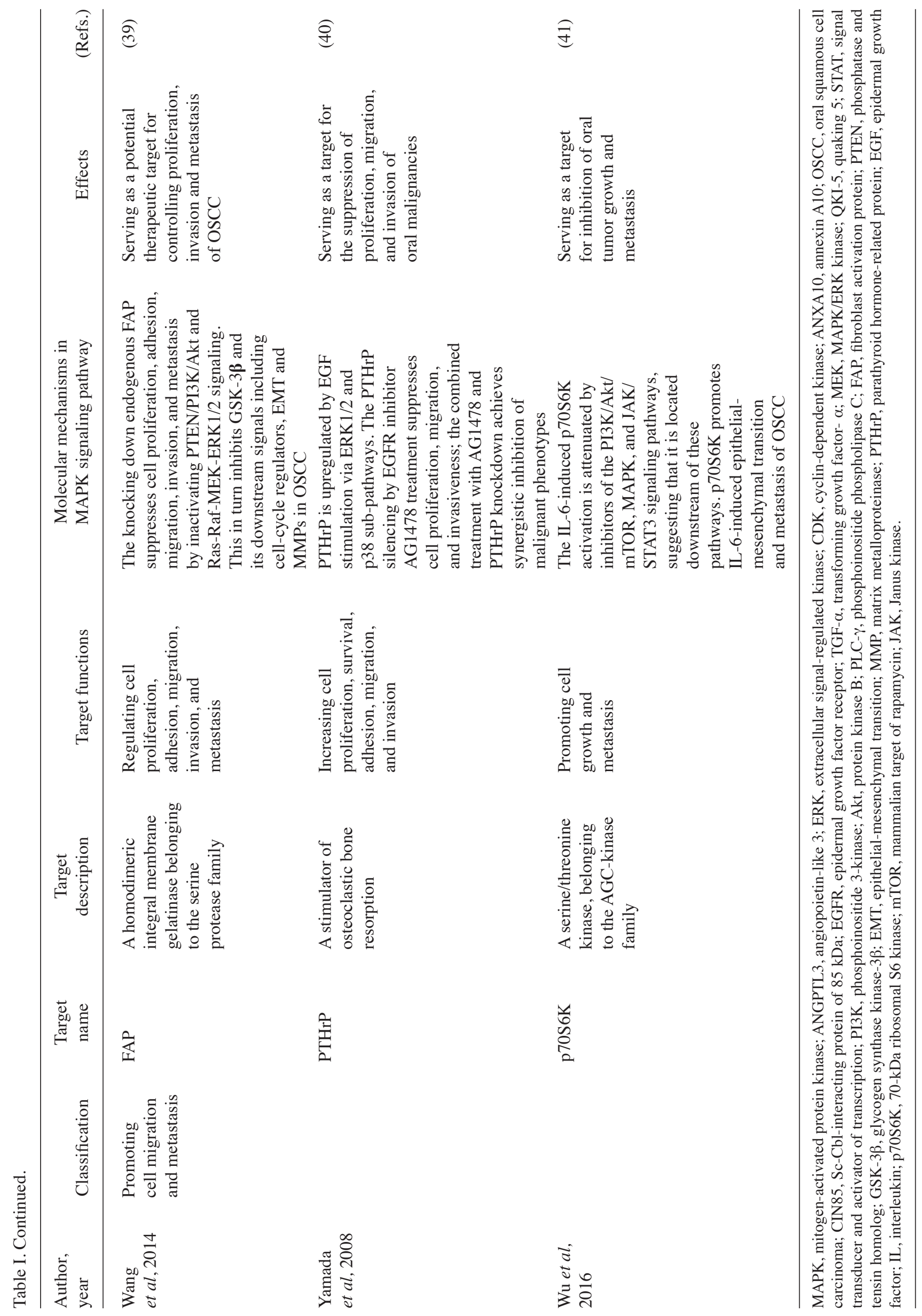




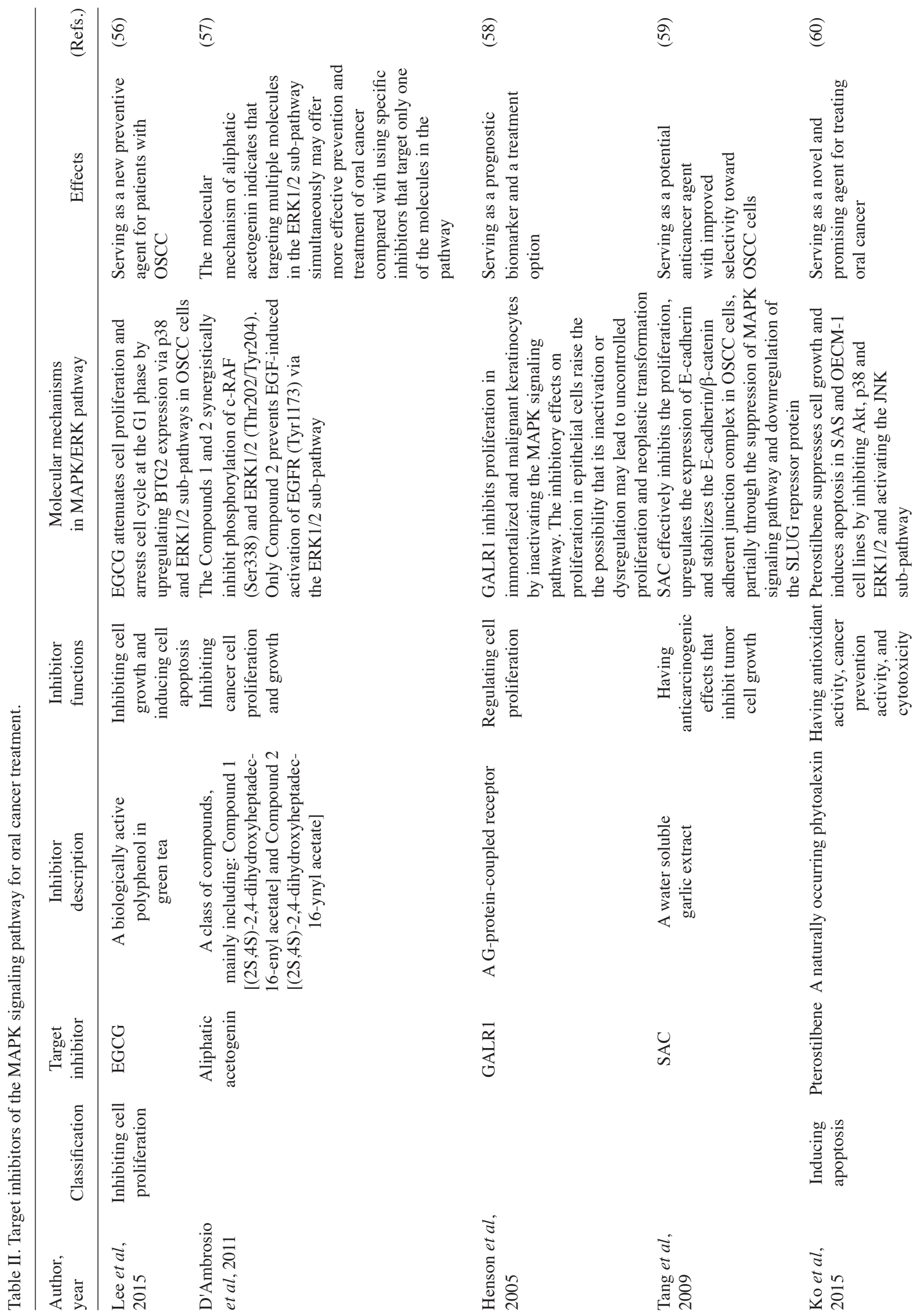




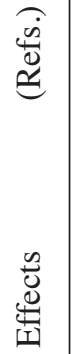

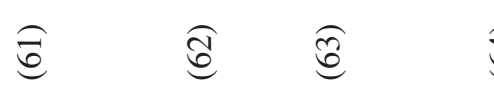

f)

\&্요

है

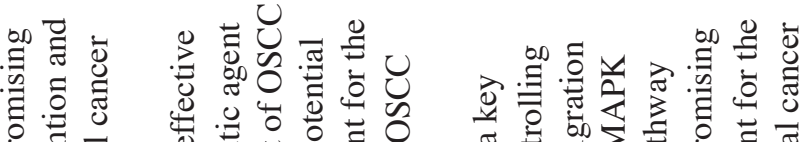

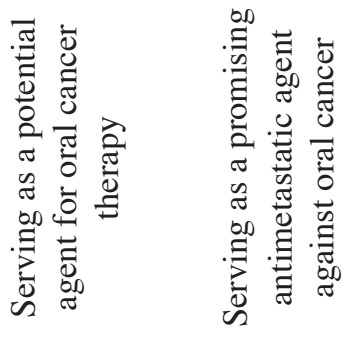

要

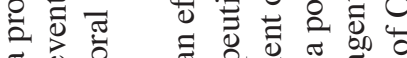

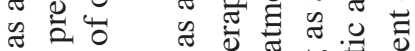

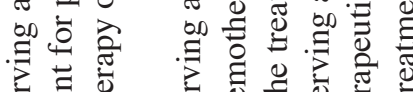

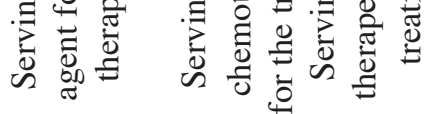

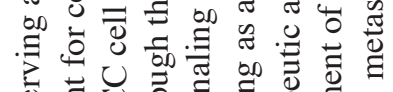

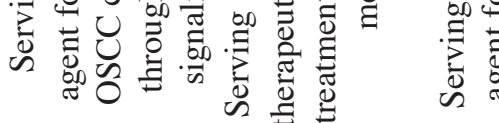

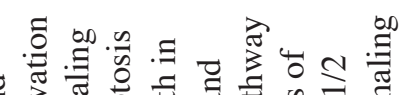

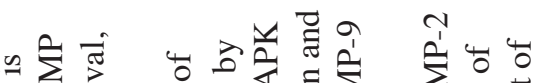

$\sum_{i}^{i}$

竞豆言

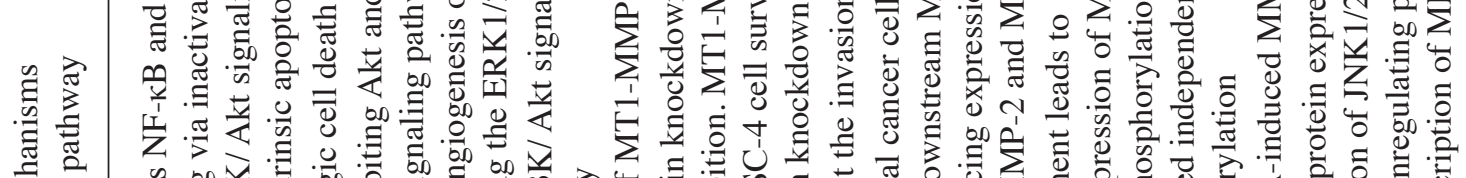

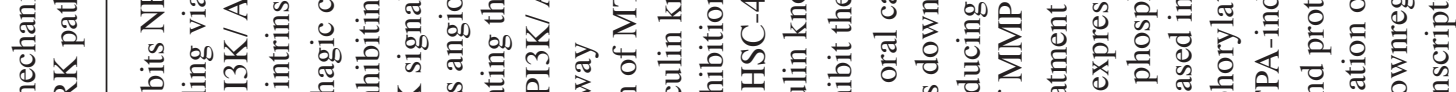

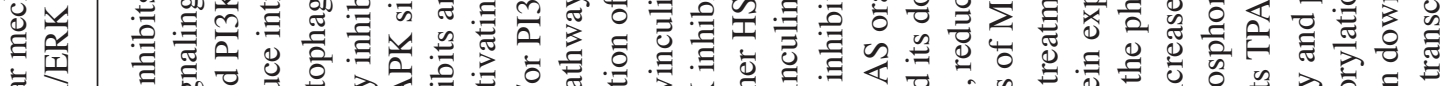

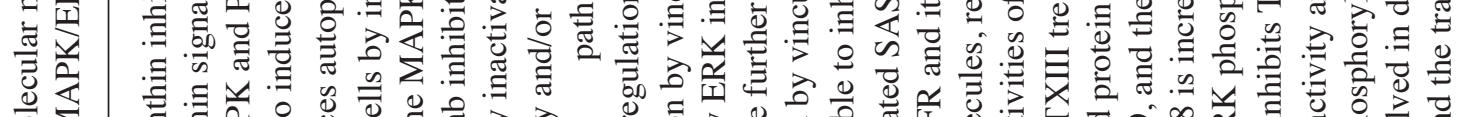
要这

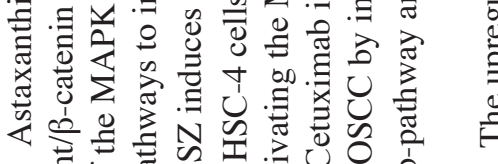

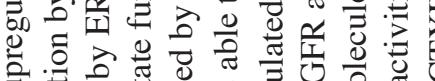

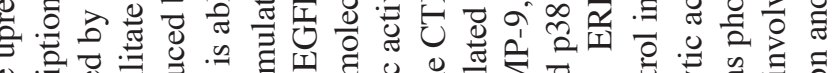

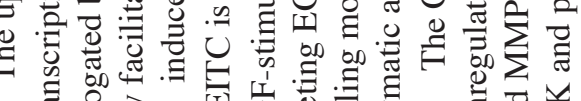

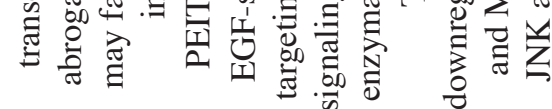

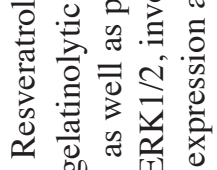

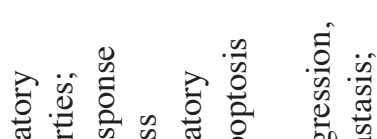

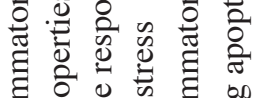

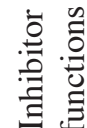

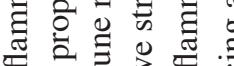

\section{:}

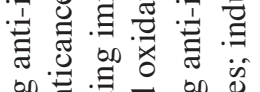

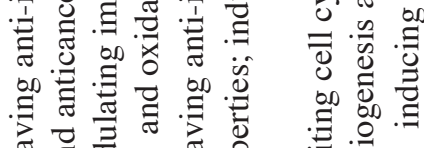

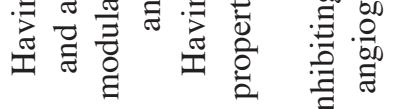

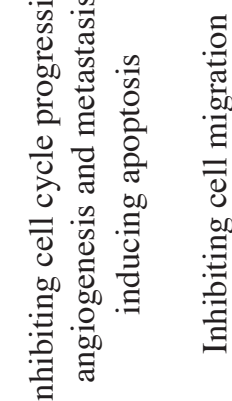

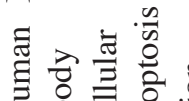

突

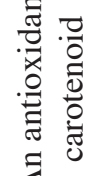

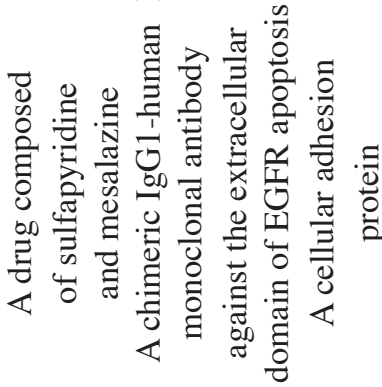

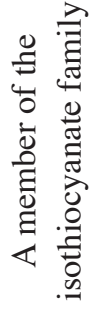

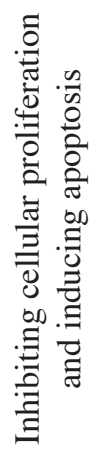

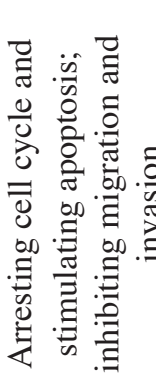
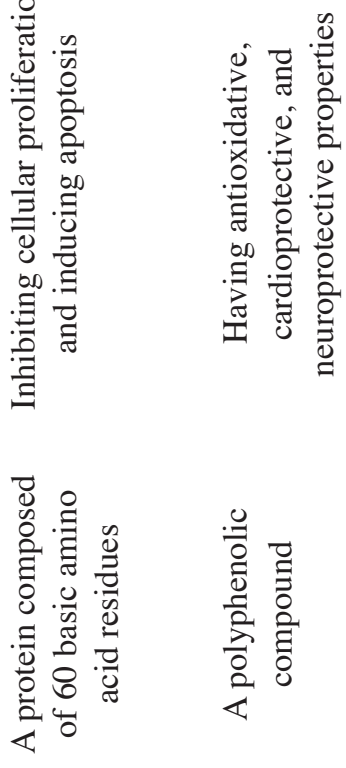

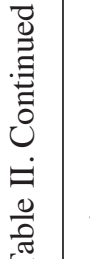

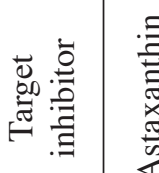

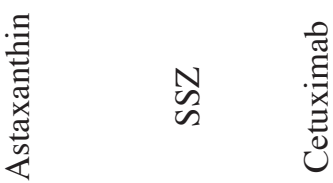

寻

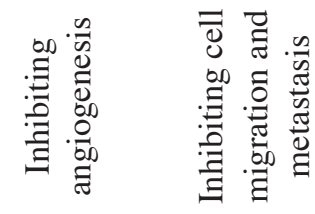

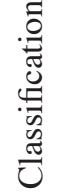<smiles>C1CCCC1</smiles>

这

䒿
草

$\frac{0}{2}$
当
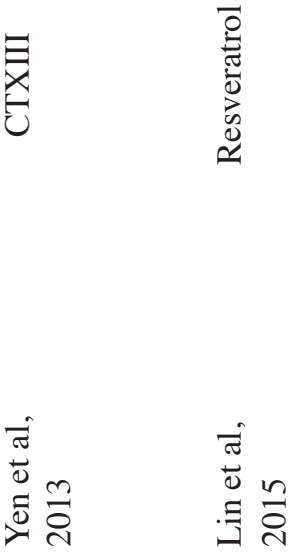

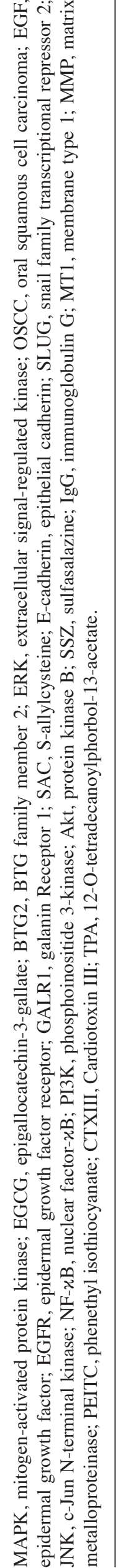


Thirdly, delivering inhibitors to specific cancer cells is difficult in certain cases (74), and regulating the MAPK signaling pathway will have direct or indirect impact on the expression of multiple downstream targets. Because the MAPK signaling pathway may cross-talk with other pathways, including the PI3K/Akt pathway, and because these other pathways are crucial for multiple aspects of cellular growth and differentiation (for example, epithelial mesenchymal transition) (75,76), inhibiting the MAPK signaling pathway may result in dysregulation of the components of other pathways. The cross regulation that exists between the pathways has partially been revealed by the control of Raf activity. As aforementioned, the activity of Raf is regulated by mutations of Ras in the MAPK signaling pathway, and/or by mutations of genes in in the PI3K/Akt pathway (including PI3K, phosphatase and tensin homolog and Akt), which may synergistically lead to abnormal activation of the MAPK pathway in oral cancer. This discovery indicates the presence of an intimate link between the MAPK and the PI3K/Akt pathways. In addition, these two pathways were reported to not only cause phosphorylation of downstream targets, but also to regulate a variety of pathways, including JAK/STAT, $\mathrm{NF}-\kappa \mathrm{B}$ and TGF- $\beta$ pathways, via ERK and Akt phosphorylation (76). In this regard, complete exploration of these pathways and their interactions should be considered in order to improve oral cancer treatment.

In addition, current clinical practice for oral cancer often discontinues a given therapy following cancer progression. However, continuous clinical practice, including use of a certain inhibitor, is desirable in certain cases because an inhibitor of one component in the MAPK signaling pathway (for example, Ras) may partially suppress another component (for example, Raf) in the same pathway, which will contribute to improved oral cancer treatment. For instance, it is helpful to add a Ras inhibitor while keeping the patient on the Raf inhibitor with which they were already being treated. This was partially confirmed when the clinical benefits of continued ERK1/2 sub-pathway inhibition following cancer progression were demonstrated (77). However, several preclinical models revealed that discontinuation of a drug based on the inhibitors or intermittent scheduling may also slow tumor growth (78). Therefore, it is necessary to carry out further clinical research to elucidate whether continuous or intermittent dosing is optimal. Proper clinical design and scheduling are important and will enable further development of effective therapies for oral cancer patients.

Finally, inhibitors that target the MAPK signaling pathway may be cytostatic and not cytotoxic (75). There would be substantial toxicity problems for cytotoxic inhibitors. To address these problems, the inhibitors should be combined with cytotoxic chemotherapeutic drugs or radiation therapy, as well as other techniques which inhibit the growth of oral cancer $(75,79)$. Such combinations are expected to improve oral cancer treatment.

In brief, the MAPK signaling pathway is complex due to the existence of multiple components, subfamilies and sub-pathways, which regulate different cellular functions, and to its interactions with other pathways which may elicit synergetic effects on normal and malignant cell growth. By further exploring the components, subfamilies and sub-pathways of the MAPK signaling pathway, as well as its interactions with other pathways and clinical practice modes, it will be possible to develop improved oral cancer treatments.

\section{Conclusions}

The present review detailed the important function of the MAPK signaling pathway in oral cancer, due to its involvement in tumor cell proliferation, differentiation, apoptosis, angiogenesis, invasion and metastasis. Based on the analysis of the individual sub-pathways and their mechanisms of action, the potential for regulation of the MAPK signaling pathway for targeted oral cancer therapy was reviewed. The challenges faced by the field and future research directions were also expanded upon. Further elucidation of the molecular components of the pathway, subfamilies, sub-pathways, interactions with other pathways and clinical practice modes will enable the development of improved oral cancer treatments.

\section{Acknowledgements}

The present study was partially supported by the National Natural Science Foundation of China (grant no. 81300841), the Health and Family Planning Commission of Hunan Province (grant no. C2016092), the Fundamental Research Funds for the Central Universities of Central South University (grant no. 2016zzts484), and the Xiangya Distinguished Doctors Program of Central South University. The authors would like to thank Dr Zhiwei Peng of the School of Mineral Processing and Bioengineering, Central South University (Changsha, China) for helpful discussion.

\section{References}

1. Grsic K, Opacic IL, Sitic S, Milkovic Perisa M, Suton P and Sarcevic B: The prognostic significance of estrogen receptor $\beta$ in head and neck squamous cell carcinoma. Oncol Lett 12: 3861-3865, 2016.

2. Peng Q, Wang Y, Quan H, Li Y and Tang Z: Oral verrucous carcinoma: From multifactorial etiology to diverse treatment regimens (Review). Int J Oncol 49: 59-73, 2016.

3. Rogers SJ, Harrington KJ, Rhys-Evans P, O-Charoenrat P and Eccles SA: Biological significance of c-erbB family oncogenes in head and neck cancer. Cancer Metastasis Rev 24: 47-69, 2005.

4. Smolensky D, Rathore K, Bourn J and Cekanova M: Inhibition of the PI3K/AKT pathway sensitizes oral squamous cell carcinoma cells to anthracycline-based chemotherapy in vitro. J Cell Biochem 118: 2615-2624, 2017.

5. Pramanik KK, Singh AK, Alam M, Kashyap T, Mishra P, Panda AK, Dey RK, Rana A, Nagini S and Mishra R: Reversion-inducing cysteine-rich protein with Kazal motifs and its regulation by glycogen synthase kinase 3 signaling in oral cancer. Tumour Biol 37: 15253-15264, 2016.

6. Sun Y, Liu WZ, Liu T, Feng X, Yang N and Zhou HF: Signaling pathway of MAPK/ERK in cell proliferation, differentiation, migration, senescence and apoptosis. J Recept Signal Transduct Res 35: 600-604, 2015.

7. Chiba T, Soeno Y, Shirako Y, Sudo H, Yagishita H, Taya Y, Kawashiri S, Okada Y and Imai K: MALT1 Inhibition of oral carcinoma cell invasion and ERK/MAPK activation. J Dent Res 95: 446-452, 2016.

8. Affolter A, Muller MF, Sommer K, Stenzinger A, Zaoui K, Lorenz K, Wolf T, Sharma S, Wolf J, Perner S, et al: Targeting irradiation-induced mitogen-activated protein kinase activation in vitro and in an ex vivo model for human head and neck cancer. Head Neck 38 (Suppl 1): E2049-E2061, 2016 
9. Kim GT, Lee SH and Kim YM: Torilis japonica extract-generated intracellular ROS induces apoptosis by reducing the mitochondrial membrane potential via regulation of the AMPK-p38 MAPK signaling pathway in HCT116 colon cancer. Int J Oncol 49: 1088-1098, 2016.

10. Song X, Wei Z and Shaikh ZA: Requirement of ER $\alpha$ and basal activities of EGFR and Src kinase in Cd-induced activation of MAPK/ERK pathway in human breast cancer MCF-7 cells. Toxicol Appl Pharmacol 287: 26-34, 2015.

11. Zheng F, Tang Q, Wu J, Zhao S, Liang Z, Li L, Wu W and Hann S: p38 $\alpha$ MAPK-mediated induction and interaction of FOXO3a and p53 contribute to the inhibited-growth and induced-apoptosis of human lung adenocarcinoma cells by berberine. J Exp Clin Cancer Res 33: 36, 2014.

12. Aguzzi A, Maggioni D, Nicolini G, Tredici G, Gaini RM and Garavello W: MAP kinase modulation in squamous cell carcinoma of the oral cavity. Anticancer Res 29: 303-308, 2009.

13. Burotto M, Chiou VL, Lee JM and Kohn EC: The MAPK pathway across different malignancies: A new perspective. Cancer 120: 3446-3456, 2014.

14. Yang SH, Sharrocks AD and Whitmarsh AJ: MAP kinase signaling cascades and transcriptional regulation. Gene 513: $1-13,2013$.

15. Krishna $M$ and Narang $H$ : The complexity of mitogen-activated protein kinases (MAPKs) made simple. Cell Mol Life Sci 65 3525-3544, 2008.

16. Reddy KB, Nabha SM and Atanaskova N: Role of MAP kinase in tumor progression and invasion. Cancer Metastasis Rev 22: 395-403, 2003

17. Cargnello M and Roux PP: Activation and function of the MAPKs and their substrates, the MAPK-activated protein kinases. Microbiol Mol Biol Rev 75: 50-83, 2011

18. Samatar AA and Poulikakos PI: Targeting RAS-ERK signalling in cancer: Promises and challenges. Nat Rev Drug Discov 13: 928-942, 2014

19. Roberts PJ and Der CJ: Targeting the Raf-MEK-ERK mitogen-activated protein kinase cascade for the treatment of cancer. Oncogene 26: 3291-3310, 2007.

20. Crane EK and Wong KK: The therapeutic promise of anti-cancer drugs against the Ras/Raf/MEK/ERK pathway. Topics Anti-Cancer Res 2: 63-94, 2013.

21. Matallanas D, Birtwistle M, Romano D, Zebisch A, Rauch J, von Kriegsheim A and Kolch W: Raf family kinases: Old dogs have learned new tricks. Genes Cancer 2: 232-260, 2011.

22. Ahearn IM, Haigis K, Bar-Sagi D and Philips MR: Regulating the regulator: Post-translational modification of RAS. Nat Rev Mol Cell Biol 13: 39-51, 2011.

23. Pratilas CA and Solit DB: Targeting the mitogen-activated protein kinase pathway: Physiological feedback and drug response. Clin Cancer Res 16: 3329-3334, 2010

24. Wortzel I and Seger R: The ERK cascade: Distinct functions within various subcellular organelles. Genes Cancer 2: 195-209, 2011.

25. Gough NR: Focus issue: Recruiting players for a game of ERK. Sci Signal 4: eg9, 2011

26. Ku NO, Azhar S and Omary MB: Keratin 8 phosphorylation by p38 kinase regulates cellular keratin filament reorganization Modulation by a keratin 1-like disease causing mutation. J Biol Chem 277: 10775-10782, 2002.

27. Gkouveris I, Nikitakis N, Karanikou M, Rassidakis G and Sklavounou A: JNK1/2 expression and modulation of STAT3 signaling in oral cancer. Oncol Lett 12: 699-706, 2016.

28. Park MK, Lee HJ, Shin J, Noh M, Kim SY and Lee CH: Novel participation of transglutaminase-2 through c-Jun N-terminal kinase activation in sphingosylphosphorylcholine-induced keratin reorganization of PANC-1 cells. Biochim Biophys Acta 1811: 1021-1029, 2011

29. Mishima K, Inoue $K$ and Hayashi Y: Overexpression of extracellular-signal regulated kinases on oral squamous cell carcinoma. Oral Oncol 38: 468-474, 2002

30. Simões AE, Rodrigues CM and Borralho PM: The MEK5/ERK5 signalling pathway in cancer: A promising novel therapeutic target. Drug Discov Today 21: 1654-1663, 2016.

31. Kato Y, Tapping RI, Huang S, Watson MH, Ulevitch RJ and Lee JD: Bmk1/Erk5 is required for cell proliferation induced by epidermal growth factor. Nature 395: 713-716, 1998.

32. Raviv Z, Kalie E and Seger R: MEK5 and ERK5 are localized in the nuclei of resting as well as stimulated cells, while MEKK2 translocates from the cytosol to the nucleus upon stimulation. J Cell Sci 117: 1773-1784, 2004.
33. Li T, Pan YW, Wang W, Abel G, Zou J, Xu L, Storm DR and Xia Z: Targeted deletion of the ERK5 MAP kinase impairs neuronal differentiation, migration, and survival during adult neurogenesis in the olfactory bulb. PLoS One 8: e61948, 2013.

34. Koyama T, Ogawara K, Kasamatsu A, Okamoto A, Kasama H, Minakawa Y, Shimada K, Yokoe H, Shiiba M, Tanzawa H and Uzawa K: ANGPTL3 is a novel biomarker as it activates ERK/MAPK pathway in oral cancer. Cancer Med 4: 759-769, 2015.

35. Shimizu T, Kasamatsu A, Yamamoto A, Koike K, Ishige S, Takatori H, Sakamoto Y, Ogawara K, Shiiba M, Tanzawa H and Uzawa K: Annexin A10 in human oral cancer: Biomarker for tumoral growth via G1/S transition by targeting MAPK signaling pathways. PLoS One 7: e45510, 2012.

36. Wakasaki T, Masuda M, Niiro H, Jabbarzadeh-Tabrizi S, Noda K, Taniyama T, Komune S and Akashi K: A Critical role of c-Cbl-Interacting protein of $85 \mathrm{kDa}$ in the development and progression of head and neck squamous cell carcinomas through the Ras-ERK pathway. Neoplasia 12: 789-796, 2010.

37. Fu X and Feng Y: QKI-5 suppresses cyclin D1 expression and proliferation of oral squamous cell carcinoma cells via MAPK signalling pathway. Int J Oral Maxillofac Surg 44: 562-567, 2015.

38. Williams MD: Integration of biomarkers including molecular targeted therapies in head and neck cancer. Head Neck Pathol 4: 62-69, 2010.

39. Wang H, Wu Q, Liu Z, Luo X, Fan Y, Liu Y, Zhang Y, Hua S, Fu Q, Zhao M, et al: Downregulation of FAP suppresses cell proliferation and metastasis through PTEN/PI3K/AKT and Ras-ERK signaling in oral squamous cell carcinoma. Cell Death Dis 5: e1155, 2014

40. Yamada T, Tsuda M, Ohba Y, Kawaguchi H, Totsuka Y and Shindoh M: PTHrP promotes malignancy of human oral cancer cell downstream of the EGFR signaling. Biochem Biophys Res Commun 368: 575-581, 2008.

41. Wu D, Cheng J, Sun G, Wu S, Li M, Gao Z, Zhai S, Li P, Su D and Wang X: p70S6K promotes IL-6-induced epithelial-mesenchymal transition and metastasis of head and neck squamous cell carcinoma. Oncotarget 7: 36539-36550, 2016.

42. Yang RW, Zeng YY, Wei WT, Cui YM, Sun HY, Cai YL, Nian XX, Hu YT, Quan YP, Jiang SL, et al: TLE3 represses colorectal cancer proliferation by inhibiting MAPK and AKT signaling pathways. J Exp Clin Cancer Res 35: 152, 2016.

43. Huang RH, Quan YJ, Chen JH, Wang TF, Xu M, Ye M, Yuan H, Zhang CJ, Liu XJ and Min ZJ: Osteopontin promotes cell migration and invasion, and inhibits apoptosis and autophagy in colorectal cancer by activating the p38 MAPK signaling pathway. Cell Physiol Biochem 41: 1851-1864, 2017.

44. Lv D, Wu H, Xing R, Shu F, Lei B, Lei C, Zhou X, Wan B, Yang Y, Zhong L, et al: HnRNP-L mediates bladder cancer progression by inhibiting apoptotic signaling and enhancing MAPK signaling pathways. Oncotarget 8: 13586-13599, 2017.

45. Chen H, Jin ZL and Xu H: MEK/ERK signaling pathway in apoptosis of SW620 cell line and inhibition effect of resveratrol. Asian Pac J Trop Med 9: 49-53, 2016.

46. Wang T, Liao Y, Sun Q, Tang H, Wang G, Zhao F and Jin Y: Upregulation of matrix metalloproteinase-9 in primary cultured rat astrocytes induced by 2-chloroethanol Via MAPK signal pathways. Front Cell Neurosci 11: 218, 2017.

47. Schafer JM, Peters DE, Morley T, Liu S, Molinolo AA, Leppla SH and Bugge TH: Efficient targeting of head and neck squamous cell carcinoma by systemic administration of a dual uPA and MMP-activated engineered anthrax toxin. PLoS One 6: e20532, 2011.

48. Munshi HG, Wu YI, Mukhopadhyay S, Ottaviano AJ, Sassano A, Koblinski JE, Platanias LC and Stack MS: Differential regulation of membrane type 1-matrix metalloproteinase activity by ERK 1/2- and p38 MAPK-modulated tissue inhibitor of metalloproteinases 2 expression controls transforming growth factor-beta1-induced pericellular collagenolysis. J Biol Chem 279: 39042-39050, 2004.

49. So KY, Kim SH, Jung KT, Lee HY and Oh SH: MAPK/JNK1 activation protects cells against cadmium-induced autophagic cell death via differential regulation of catalase and heme oxygenase-1 in oral cancer cells. Toxicol Appl Pharmacol 332: 81-91, 2017.

50. Cossa G, Gatti L, Cassinelli G, Lanzi C, Zaffaroni N and Perego P: Modulation of sensitivity to antitumor agents by targeting the MAPK survival pathway. Curr Pharm Des 19: $883-894,2013$ 
51. De Luca A, Maiello MR, D'Alessio A, Pergameno $M$ and Normanno N: The RAS/RAF/MEK/ERK and the PI3K/AKT signaling pathways: Role in cancer pathogenesis and implications for therapeutic approaches. Expert Opin Ther Targets 16 (Suppl 2): S17-S27, 2012.

52. Wang Y, Liu J, Cui F, Xing L, Wang J, Yan X and Zhang X: ERK and p38 MAPK signaling pathways are involved in ochratoxin A-induced G2 phase arrest in human gastric epithelium cells. Toxicol Lett 209: 186-192, 2012.

53. Ahn HJ, Kim G and Park KS: Ell3 stimulates proliferation, drug resistance, and cancer stem cell properties of breast cancer cells via a MEK/ERK-dependent signaling pathway. Biochem Biophys Res Commun 437: 557-564, 2013.

54. Neuzillet C, Hammel P, Tijeras-Raballand A, Couvelard A and Raymond E: Targeting the Ras-ERK pathway in pancreatic adenocarcinoma. Cancer Metastasis Rev 32: 147-162, 2013.

55. Ahmeda TA, Hayslip J and Leggas M: Simvastatin interacts synergistically with tipifarnib to induce apoptosis in leukemia cells through the disruption of RAS membrane localization and ERK pathway inhibition. Leuk Res 38: 1350-1357, 2014.

56. Lee JC, Chung LC, Chen YJ, Feng TH, Chen WT and Juang HH: Upregulation of B-cell translocation gene 2 by epigallocatechin-3-gallate via p38 and ERK signaling blocks cell proliferation in human oral squamous cell carcinoma cells. Cancer Lett 360: 310-318, 2015.

57. D'Ambrosio SM, Han C, Pan L, Kinghorn AD and Ding $\mathrm{H}$ Aliphatic acetogenin constituents of avocado fruits inhibit human oral cancer cell proliferation by targeting the EGFR/RAS/RAF/MEK/ERK1/2 pathway. Biochem Biophys Res Commun 409: 465-469, 2011.

58. Henson BS, Neubig RR, Jang I, Ogawa T, Zhang Z, Carey TE and D'Silva NJ: Galanin receptor 1 has anti-proliferative effects in oral squamous cell carcinoma. J Biol Chem 280: 22564-22571, 2005.

59. Tang FY, Chiang EP, Chung JG, Lee HZ and Hsu CY: S-Allylcysteine modulates the expression of E-cadherin and inhibits the malignant progression of human oral cancer. J Nutr Biochem 20: 1013-1020, 2009.

60. Ko CP, Lin CW, Chen MK, Yang SF, Chiou HL and Hsieh MJ: Pterostilbene induce autophagy on human oral cancer cells through modulation of Akt and mitogen-activated protein kinase pathway. Oral Oncol 51: 593-601, 2015.

61. Kavitha K, Kowshik J, Kishore TK, Baba AB and Nagini S: Astaxanthin inhibits NF- $\mathrm{BB}$ and $\mathrm{Wnt} / \beta$-catenin signaling pathways via inactivation of Erk/MAPK and PI3K/Akt to induce intrinsic apoptosis in a hamster model of oral cancer. Biochim Biophys Acta 1830: 4433-4444, 2013.

62. Han HY, Kim H, Jeong SH, Lim DS and Ryu MH: Sulfasalazine induces autophagic cell death in oral cancer cells via Akt and ERK pathways. Asian Pac J Cancer Prev 15 6939-6944, 2014.

63. Psyrri A, Lee JW, Pectasides E, Vassilakopoulou M Kosmidis EK, Burtness BA, Rimm DL, Wanebo HJ and Forastiere AA: Prognostic biomarkers in phase II trial of cetuximab-containing induction and chemoradiation in resectable HNSCC: Eastern cooperative oncology group E2303. Clin Cancer Res 20: 3023-3032, 2014.

64. Yoshimoto T, Takino T, Li Z, Domoto T and Sato H: Vinculin negatively regulates transcription of MT1-MMP through MEK/ERK pathway. Biochem Biophys Res Commun 455: 251-255, 2014

65. Chen HJ, Lin CM, Lee CY, Shih NC, Amagaya S, Lin YC and Yang JS: Phenethyl isothiocyanate suppresses EGF-stimulated SAS human oral squamous carcinoma cell invasion by targeting EGF receptor signaling. Int J Oncol 43: 629-637, 2013.
66. Yen CY, Liang SS, Han LY, Chou HL, Chou CK, Lin SR and Chiu CC: Cardiotoxin III inhibits proliferation and migration of oral cancer cells through MAPK and MMP signaling. Scientific World Journal 2013: 650946, 2013.

67. Lin FY, Hsieh YH, Yang SF, Chen CT, Tang CH, Chou MY, Chuang YT, Lin CW and Chen MK: Resveratrol suppresses TPA-induced matrix metalloproteinase- 9 expression through the inhibition of MAPK pathways in oral cancer cells. J Oral Pathol Med 44: 699-706, 2015.

68. Jänne PA, Shaw AT, Pereira JR, Jeannin G, Vansteenkiste J, Barrios C, Franke FA, Grinsted L, Zazulina V, Smith P, et al: Selumetinib plus docetaxel for KRAS-mutant advanced non-small-cell lung cancer: A randomised, multicentre, placebo-controlled, phase 2 study. Lancet Oncol 14: 38-47, 2013.

69. Grisham RN, Iyer G, Garg K, DeLair D, Hyman DM, Zhou Q, Iasonos A, Berger MF, Dao F, Spriggs DR, et al: BRAF mutation is associated with early stage disease and improved outcome in patients with low-grade serous ovarian cancer. Cancer 119: 548-554, 2013.

70. Das N, Majumder J and DasGupta UB: Ras gene mutations in oral cancer in eastern India. Oral Oncol 36: 76-80, 2000.

71. Martini M, De Santis MC, Braccini L, Gulluni F and Hirsch E: PI3K/AKT signaling pathway and cancer: An updated review. Ann Med 46: 372-383, 2014.

72. Collisson EA, De A, Suzuki H, Gambhir SS and Kolodney MS: Treatment of metastatic melanoma with an orally available inhibitor of the Ras-Raf-MAPK cascade. Cancer Res 63: 5669-5673, 2003

73. Wan PT, Garnett MJ, Roe SM, Lee S, Niculescu-Duvaz D, Good VM, Jones CM, Marshall CJ, Springer CJ, Barford D, et al: Mechanism of activation of the RAF-ERK signaling pathway by oncogenic mutations of B-RAF. Cell 116: 855-867, 2004.

74. Antipina MN, Kiryukhin MV, Skirtach AG and Sukhorukov GB: Micropackaging via layer-by-layer assembly: Microcapsules and microchamber arrays. Int Mater Rev 59: 224-244, 2014.

75. Steelman LS, Chappell WH, Abrams SL, Kempf RC, Long J, Laidler P, Mijatovic S, Maksimovic-Ivanic D, Stivala F, Mazzarino MC, et al: Roles of the Raf/MEK/ERK and $\mathrm{PI} 3 \mathrm{~K} / \mathrm{PTEN} / \mathrm{Akt} / \mathrm{mTOR}$ pathways in controlling growth and sensitivity to therapy-implications for cancer and aging. Aging (Albany NY) 3: 192-222, 2011.

76. Steelman LS, Abrams SL, Whelan J, Bertrand FE, Ludwig DE, Bäsecke J, Libra M, Stivala F, Milella M, Tafuri A, et al: Contributions of the Raf/MEK/ERK,PI3K/PTEN/Akt/mTOR and JAK/STAT pathways to leukemia. Leukemia 22: 686-707, 2008.

77. Carlino MS, Gowrishankar K, Saunders CA, Pupo GM, Snoyman S, Zhang XD, Saw R, Becker TM, Kefford RF, Long GV and Rizos H: Antiproliferative effects of continued mitogen-activated protein kinase pathway inhibition following acquired resistance to BRAF and/or MEK inhibition in melanoma. Mol Cancer Ther 12: 1332-1342, 2013.

78. Das Thakur M, Salangsang F, Landman AS, Sellers WR, Pryer NK, Levesque MP, Dummer R, McMahon M and Stuart DD: Modelling vemurafenib resistance in melanoma reveals a strategy to forestall drug resistance. Nature 494: $251-255,2013$.

79. Imparato $G$, Urciuolo $F$ and Netti PA: In vitro three-dimensional models in cancer research: A review. Int Mater Rev 60: 297-311, 2015. 